The Journal of $\mathbf{N}_{\text {ondinear }} \mathbf{S}_{\text {cience and }}$ Applications

http://www.tjnsa.com

\title{
STABILIZABILITY OF A CLASS OF NONLINEAR SYSTEMS USING HYBRID CONTROLLERS
}

\author{
XINZHI LIU ${ }^{1 *}$ AND PETER STECHLINSKI ${ }^{1}$
}

\begin{abstract}
This paper develops hybrid control strategies for stabilizing a class of nonlinear systems. Common Lyapunov functions and switched Lyapunov functions are used to establish easily verifiable criteria for the stabilizability of weakly nonlinear systems under switched and impulsive control. Three types of controller switching rules are studied: time-dependent (synchronous), statedependent (asynchronous) and average dwell-time satisfying. Conditions are developed for stabilizability under arbitrary switching, as well as less strict conditions for prespecified switching rules. Examples are given, with simulations, to illustrate the theorems developed.
\end{abstract}

\section{INTRODUCTION}

In recent years there has been a wide interest in the study of hybrid dynamical systems, which combine continuous/discrete dynamics with logic-based switching [22]. These systems evolve according to mode-dependent continuous/discrete dynamics, and, triggered by threshold events, experience abrupt changes between modes [22]. A switched system, which is a type of hybrid system, often arises in two contexts [5]: The first is when there is an abrupt change in the dynamics of a natural system, which could be due to, for example, environmental factors. The second context is when a continuous system is stabilized using switching controllers. The latter is an interesting and important problem to study, as a system that cannot be stabilized by a continuous controller may be stabilizable by a switching controller [15].

Date: Received: 1 October 2010; Revised: 29 October 2010.

* Corresponding author

(C) 2010 N.A.G.

2000 Mathematics Subject Classification. Primary 93C30; Secondary 37C75, 93D15.

Key words and phrases. Hybrid systems; Switched systems; Stabilizability; Switched control; Impulsive control; Synchronous switching; State-dependent switching; Dwell-time switching. 
Many important and interesting problems, found in areas such as computer science, control engineering, and applied mathematics, can be modelled naturally by hybrid systems [7]. Practical applications of switched systems include many diverse areas, for example, mechanical systems, the automotive industry, air traffic control, robotics, intelligent vehicle/highway systems, robotics, integrated circuit design, multimedia, manufacturing, power electronics, switched-capacitor networks, chaos generators, computer disk drives, automotive engine management, high-level flexible manufacturing systems, job scheduling, interconnected power systems, and chemical processes [5, 7, 8, 15]. Switched systems have been shown to exhibit interesting behaviour, such as the switched and impulsive control of unstable subsystems that leads to a stable system $[8,9]$, and the instability of a switched system comprised solely of stable subsystems [15].

The majority of the work in the switched systems literature pertains to continuous and discrete switched systems, but these systems fail to properly model real world systems which exhibit an impulsive effect at switching points [8]. There are currently few reports in the literature that study the stability of switched and impulsive systems [9]. Examples of switched systems with impulsive effects at switching instances include biological neural networks, bursting rhythm models in pathology, and optimal control in economics [8]. Another important reason for studying switched and impulsive systems stems from a control point of view: traditional control is not always adequate for the stabilization of complex dynamic systems, but switched control may be combined with impulsive control to increase performance [9].

Some standard techniques to study the stability of switched systems, which is important in many applications, are common and switched Lyapunov functions $[2,3,5,20]$, and switched invariance principles [1, 10, 11]. Switched systems with subsystems that are triangularizable have been studied [17], linear switched systems with commuting subsystems have been analyzed [19], and switched systems with average dwell-time satisfying switching rules have been considered [11, 12, 22]. The control of discrete switched systems has been investigated [4], the stabilization of nonlinear switched systems using feedback control and control Lyapunov functions has been studied [18], and the instability of switched systems under arbitrary switching has been analyzed [21]. For a general overview of hybrid and switched systems, as well as a review of current literature in the area, see $[5,6,14,15,22,23,24]$.

Motivated by the extensive practical applications mentioned earlier, this paper aims to study weakly nonlinear impulsive and switched systems. This paper's objective is to establish new and easily verifiable criteria for the stabilization of impulsive and weakly nonlinear switched systems under both asynchronous and synchronous switching. Secondly, this paper aims to extend current results on switched and impulsive systems with dwell-time satisfying switching rules to include nonlinear switched control and nonlinear impulsive control. In doing this, results from [7] and [9] are extended by studying the stabilization of weakly nonlinear switched and impulsive systems under asynchronous, synchronous, and dwell-time satisfying switching rules. 
This paper is organized as follows: Section 2 gives a mathematical formulation of the problem. In Section 3, asynchronous switching is introduced and studied. Criteria are given for the stabilizability of switched systems under a prespecified asynchronous switching rule. Further, some stricter conditions are given for stabilizability under arbitrary asynchronous switching. Section 4 investigates synchronous switching, establishing criteria for stabilizability under both arbitrary synchronous switching as well as a prespecified synchronous switching rule. Average dwell-time satisfying switching is studied in Section 5. Examples are given in Section 6, with simulations using Matlab, to illustrate the various theorems established in the paper. Finally, some conclusions are made and future directions are given in Section 7.

\section{Problem Formulation}

Let $\mathbb{R}_{+}$denote the set of nonnegative real numbers and let $\mathbb{R}^{n}$ denote the Euclidean space of n-dimensions with Euclidean norm $\|\cdot\|$. Let $\lambda_{\max }[Q]\left(\lambda_{\min }[Q]\right)$ denote the maximum (minimum) eigenvalue of a symmetric matrix $Q$, respectively. Consider the following control system:

$$
\dot{x}(t)=A x(t)+F(t, x)+B u(t)+C v(t),
$$

where $x \in \mathbb{R}^{n}$ is the state vector, $u \in \mathbb{R}^{h}, v \in \mathbb{R}^{h}$ are controllers, $t \in \mathbb{R}_{+}, A, B$ and $C$ are constant matrices of corresponding dimensions, and $F(t, x)$ is a continuous vector-valued function such that $F(t, 0) \equiv 0$ for all $t \geq t_{0}$.

Extending [7], assume that there are a collection of $m$ basic state feedback controllers: $u_{L_{1}}(t)=L_{1} x(t), \ldots, u_{L_{m}}(t)=L_{m} x(t)$, where $L_{i}$ are constant control gain matrices of corresponding dimension, and a collection of $m$ nonlinear state feedback controllers: $u_{J_{1}}(t)=J_{1}(t, x), \ldots, u_{J_{m}}(t)=J_{m}(t, x)$, where $J_{i}(t, x)$ are piecewise continuous vector-valued functions such that $J_{i}(t, 0) \equiv 0$ for all $t \geq t_{0}$. Further, assume that there are a collection of $m$ impulsive controllers: $v_{M_{1}}(t)=$ $M_{1} x(t) \delta\left(t-t_{k}\right), \ldots, v_{M_{m}}(t)=M_{m} x(t) \delta\left(t-t_{k}\right)$, where $M_{i}$ are constant control gain matrices and $\delta(t)$ is the Dirac delta generalized function. Finally, assume that there are a collection of $m$ nonlinear impulsive controllers: $v_{Q_{1}}(t)=Q_{1}(t, x) \delta(t-$ $\left.t_{k}\right), \ldots, v_{Q_{m}}(t)=Q_{m}(t, x) \delta\left(t-t_{k}\right)$, where $Q_{i}(t, x)$ are piecewise continuous vectorvalued functions such that $Q_{i}(t, 0) \equiv 0$ for all $t \geq t_{0}$. Following the procedure of [8], incorporate these controllers into system (2.1) by constructing the control inputs as follows:

$$
\begin{gathered}
u(t)=\sum_{k=1}^{\infty} L_{i_{k}} x(t) l_{k}(t)+\sum_{k=1}^{\infty} J_{i_{k}}(t, x) l_{k}(t), \\
\text { where } l_{k}(t):= \begin{cases}1 & \text { if } t \in\left(t_{k-1}, t_{k}\right], \\
0 & \text { otherwise, }\end{cases} \\
v(t)=\sum_{k=1}^{\infty} M_{i_{k}} x(t) \delta\left(t-t_{k}\right)+\sum_{k=1}^{\infty} Q_{i_{k}}(t, x) \delta\left(t-t_{k}\right),
\end{gathered}
$$

with discontinuity points $t_{1}<\ldots<t_{k}<\ldots \rightarrow \infty$ as $k \rightarrow \infty$ and where $i_{k} \in\{1,2, \ldots, m\}$. Following [8], note that based on the definition of $l_{k}(t)$, it is 
apparent that the controller $u(t)$ switches its value at every time $t=t_{k}$, hence $u(t)$ is a switching controller. To interpret $v(t)$, observe that $x\left(t_{k}+h\right)-x\left(t_{k}\right)=$ $\int_{t_{k}}^{t_{k}+h}[A x(s)+F(t, s)+B u(s)+C v(s)] d s$. As $h \rightarrow 0^{+}, x\left(t_{k}^{+}\right)-x\left(t_{k}\right)=C M_{i_{k}} x\left(t_{k}\right)+$ $C Q_{i_{k}}\left(t_{k}, x\left(t_{k}\right)\right)$, where $x\left(t_{k}^{+}\right):=\lim _{h \rightarrow 0^{+}} x\left(t_{k}+h\right)$. That is, there is a sudden jump in the state of the system at each $t=t_{k}$, hence $v(t)$ is an impulsive controller.

For $t_{0} \in \mathbb{R}_{+}$and $x_{0} \in \mathbb{R}^{n}$, system (2.1) can be rewritten as a switched impulsive system:

$$
\left\{\begin{aligned}
\dot{x} & =\left(A+B L_{i_{k}}\right) x+F(t, x)+B J_{i_{k}}(t, x), & & t \in\left(t_{k-1}, t_{k}\right], \\
\Delta x & =C M_{i_{k}} x+C Q_{i_{k}}(t, x), & & t=t_{k}, \\
x\left(t_{0}^{+}\right) & =x_{0}, & & k=1,2, \ldots
\end{aligned}\right.
$$

where $\Delta x:=x\left(t_{k}^{+}\right)-x\left(t_{k}\right)$ and $i_{k} \in\{1,2, \ldots, m\}$ follows a switching rule

$$
\sigma: \mathbb{R}_{+} \times \mathbb{R}^{n} \rightarrow\{1,2, \ldots, m\}
$$

where $\sigma$ is a piecewise continuous function assumed to be left-continuous. For example, a simple switching rule construction is $\sigma:\left(t_{k-1}, t_{k}\right] \rightarrow\{1,2, \ldots, m\}$, $k=1,2, \ldots$, which has switch times $t=t_{k}$ where system (2.2) undergoes an impulsive effect and a switch in the dynamics governing the system. That is, $i=\sigma(t)$ for $t \in\left(t_{k-1}, t_{k}\right]$ and $i=\sigma\left(t_{k}^{+}\right)$immediately after the switch and impulsive time $t_{k}$. See Figure 1 for an illustration of this simple switching rule. Under the general construction (2.3), it is possible the switching rule is time-dependent, state-dependent, or a combination of both. Note that if the switching times are not based on the state of the system, it is possible the switching rule is still state-dependent (see Section 4).

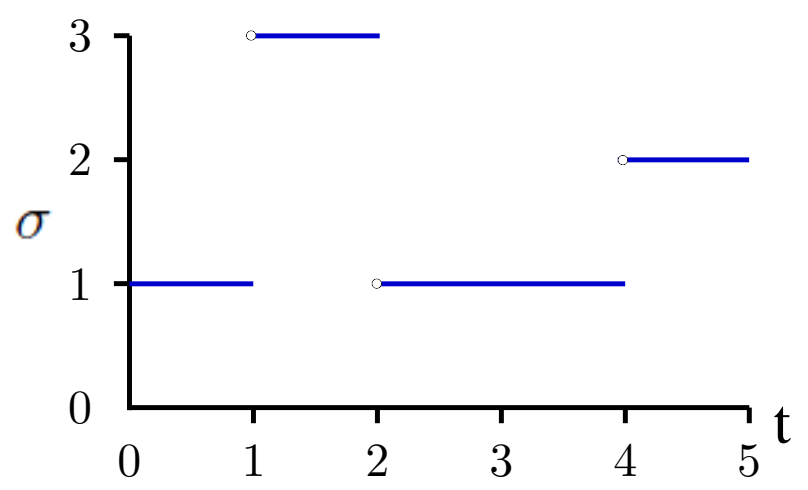

FiguRE 1. Example of a switching rule $\sigma$ with switch times $t_{k}=1,2,4$.

Given a set of constant control matrices $\left\{L_{i}\right\},\left\{M_{i}\right\}$, and a set of nonlinear controllers $\left\{J_{i}(t, x)\right\},\left\{Q_{i}(t, x)\right\}$, the objective is to find a switching control time sequence $\left\{t_{k}\right\}$, and a switching rule $\sigma$ such that the trivial solution of system (2.2) is globally asymptotically stable. The trivial solution is said to be stable for a switching rule $\sigma$ if, for any $\epsilon>0$, there exists a $\delta>0$ such that $\left\|x\left(t_{0}\right)\right\|<\delta$ implies $\|x(t)\|<\epsilon$ for any $t \geq t_{0}$. The trivial solution is asymptotically stable if it is stable and there exists a $\beta>0$ such that $\left\|x\left(t_{0}\right)\right\|<\beta$ implies $\lim _{t \rightarrow \infty} x(t)=$ 
0 . Further, the trivial solution is exponentially stable if there exist constants $\alpha, \gamma, C>0$ such that if $\left\|x\left(t_{0}\right)\right\|<\alpha$ then $\|x(t)\|<C\left\|x\left(t_{0}\right)\right\| e^{-\gamma\left(t-t_{0}\right)}$ for any $t \geq$ $t_{0}$. Finally, the trivial solution is globally asymptotically (exponentially) stable if it is asymptotically (exponentially) stable and $\beta(\alpha)$ is arbitrary, respectively. Note that exponential stability implies asymptotic stability.

\section{Asynchronous CONTROLler SWitching}

Consider system (2.2) and suppose that the switching rule takes the following form:

$$
\sigma(x(t)): \mathbb{R}^{n} \rightarrow\{1,2, \ldots, m\},
$$

which is called asynchronous controller switching [7]. This switching rule implicitly defines the switch times $t_{k}$ when the state crosses a switching threshold that is based on the state of the system. Under this construction the switched system is,

$$
\left\{\begin{aligned}
\dot{x} & =\left(A+B L_{i_{k}}\right) x+F(t, x)+B J_{i_{k}}(t, x), & & t \in\left(t_{k-1}, t_{k}\right], \\
\Delta x & =C M_{i_{k}} x+C Q_{i_{k}}(t, x), & & t=t_{k}, \\
x\left(t_{0}^{+}\right) & =x_{0}, & & k=1,2, \ldots,
\end{aligned}\right.
$$

where $i_{k} \in\{1,2, \ldots, m\}$ follows an asynchronous switching rule $\sigma(x)$. When a certain state threshold is reached (corresponding to a switching time $t_{k}$ ), the switching rule $\sigma(x)$ selects the next subsystem to switch to based on the system state $x\left(t_{k}\right)$. Extending some results from [7], consider the following stabilizability theorem for a prespecified asynchronous switching rule.

Theorem 3.1. Assume that there exists matrices $G, H_{i}, K_{i}=K_{i}^{T}$, positive definite matrix $P=P^{T}$, and constants $\mu_{i}$ which satisfy,

$$
\begin{gathered}
2 F^{T}(t, x) P x \leq x^{T} G x, \\
2 J_{i}^{T}(t, x) B^{T} P x \leq x^{T} H_{i} x, \\
2 x^{T}\left(I+C M_{i}\right)^{T} P C Q_{i}(t, x)+Q_{i}^{T}(t, x) C^{T} P C Q_{i}(t, x) \leq x^{T} K_{i} x, \\
\lambda_{\max }\left[P^{-1}\left(\left(I+C M_{i}\right)^{T} P\left(I+C M_{i}\right)+K_{i}\right)\right] \leq \mu_{i},
\end{gathered}
$$

for $x \in \mathbb{R}^{n}, t \geq t_{0}$, and $i=1, \ldots, m$. Assume that there exists constants $\tau_{1} \geq$ $0, \ldots, \tau_{m} \geq 0$ and $D>0$ such that $\sum_{i=1}^{m} \tau_{i}=1, \sum_{i=1}^{m} \tau_{i} \Upsilon_{i}<0$, where $\Upsilon_{i}=$ $\left(A+B L_{i}\right)^{T} P+P\left(A+B L_{i}\right)+H_{i}+G$, and $\sum_{k=1}^{\infty}\left(\mu_{i_{k}}-1\right)<D$. Define the switching regions $\Omega_{i}=\left\{x \in \mathbb{R}^{n}: x^{T} \Upsilon_{i} x \leq x^{T} \Upsilon_{j} x, j=1,2, \ldots, m\right\}$, define $\partial \Omega_{i}$ to be the boundary of $\Omega_{i}$, and define the minimum rule $f(x)=\arg \min _{i} x^{T} \Upsilon_{i} x$. If the asynchronous switching rule follows the algorithm,

(i) set $\sigma=f\left(x\left(t_{0}\right)\right)$ for $t=t_{0}$;

(ii) maintain $\sigma=i$ in the region $\Omega_{i}$ until $x\left(t_{1}\right) \in \partial \Omega_{i}$ at $t=t_{1}$;

(iii) apply an impulse and let $t_{0}=t_{1}^{+}$then go to step (3.7);

then the trivial solution of system (3.2) is globally exponentially stable. 
Proof. Following [7], consider the common Lyapunov function $V=x^{T} P x$ and take the time-derivative along solutions of (3.2) for $t \in\left(t_{k-1}, t_{k}\right]$,

$$
\begin{aligned}
\dot{V}(x(t))= & {\left[\left(A+B L_{i_{k}}\right) x+F(t, x)+B J_{i_{k}}(t, x)\right]^{T} P x } \\
& +x^{T} P\left[\left(A+B L_{i_{k}}\right) x+F(t, x)+B J_{i_{k}}(t, x)\right], \\
= & x^{T}\left[\left(A+B L_{i_{k}}\right)^{T} P+P\left(A+B L_{i_{k}}\right)\right] x+F^{T}(t, x) P x+x^{T} P F(t, x) \\
& +J_{i_{k}}^{T}(t, x) B^{T} P x+x^{T} P B J_{i_{k}}(t, x), \\
\leq & x^{T}\left[\left(A+B L_{i_{k}}\right)^{T} P+P\left(A+B L_{i_{k}}\right)\right] x+x^{T} H_{i_{k}} x+x^{T} G x \\
= & x^{T} \Upsilon_{i_{k}} x .
\end{aligned}
$$

The condition $\tau_{1} x^{T} \Upsilon_{1} x+\ldots+\tau_{m} x^{T} \Upsilon_{m} x<0$ implies that for any $x \in \mathbb{R}^{n} \backslash\{0\}$, there exists an index $i \in\{1, \ldots, m\}$ such that $x^{T} \Upsilon_{i} x<0$. As in [7], define $\alpha(x):=\min _{\forall i} x^{T} \Upsilon_{i} x$ for $x \neq 0$ and $\alpha_{0}:=\max _{x:\|x\|=1} \alpha(x)$ which is achieved on this compact set since $\alpha(x)$ is continuous. Since there exists an $i$ for any $x \in \mathbb{R}^{n} \backslash\{0\}$ such that $x^{T} \Upsilon_{i} x<0$, it follows that $\alpha(x)<0$ for all $x \neq 0$, and hence $\alpha_{0}<0$. Since the switching rule follows (3.7)-(3.9), it follows from (3.10) that, for $t \neq t_{k}$,

$$
\dot{V}(x(t)) \leq \alpha_{0}\|x(t)\|^{2} \leq \frac{\alpha_{0}}{\lambda_{\min }[P]} x^{T} P x=-\epsilon V(x(t)),
$$

with $\epsilon=-\alpha_{0} / \lambda_{\min }[P]>0$. Suppose that, without loss of generality, the switch and impulsive times given by the asynchronous rule $\sigma(x)$ are $t_{k}, k=1,2, \ldots$, with $t_{k}>t_{k-1}$, and $t_{k} \rightarrow \infty$ as $k \rightarrow \infty$, then for $t \in\left(t_{k-1}, t_{k}\right]$,

$$
V(x(t)) \leq V\left(x\left(t_{k}^{+}\right)\right) \exp \left[-\epsilon\left(t-t_{k-1}\right)\right] .
$$

Immediately after the time $t=t_{k}$ an impulse is applied:

$$
\begin{aligned}
V\left(x\left(t_{k}^{+}\right)\right)= & x^{T}\left(t_{k}^{+}\right) P x\left(t_{k}^{+}\right) \\
= & {\left[\left(I+C M_{i_{k}}\right) x\left(t_{k}\right)+C Q_{i_{k}}\left(t_{k}, x\left(t_{k}\right)\right)\right]^{T} P\left[\left(I+C M_{i_{k}}\right) x\left(t_{k}\right)\right.} \\
& \left.+C Q_{i_{k}}\left(t_{k}, x\left(t_{k}\right)\right)\right] \\
= & x^{T}\left(t_{k}\right)\left(I+C M_{i_{k}}\right)^{T} P\left(I+C M_{i_{k}}\right) x\left(t_{k}\right) \\
& +Q_{i_{k}}^{T}\left(t_{k}, x\left(t_{k}\right)\right) C^{T} P\left(I+C M_{i_{k}}\right) x\left(t_{k}\right) \\
& +x^{T}\left(t_{k}\right)\left(I+C M_{i_{k}}\right)^{T} P C Q_{i_{k}}\left(t_{k}, x\left(t_{k}\right)\right) \\
& +Q_{i_{k}}^{T}\left(t_{k}, x\left(t_{k}\right)\right) C^{T} P C Q_{i_{k}}\left(t_{k}, x\left(t_{k}\right)\right), \\
\leq & x^{T}\left(t_{k}\right)\left[\left(I+C M_{i_{k}}\right)^{T} P\left(I+C M_{i_{k}}\right)+K_{i_{k}}\right] x\left(t_{k}\right), \\
\leq & \lambda_{\max }\left[P^{-1}\left(\left(I+C M_{i_{k}}\right)^{T} P\left(I+C M_{i_{k}}\right)+K_{i_{k}}\right)\right] x^{T}\left(t_{k}\right) P x\left(t_{k}\right), \\
\leq & \mu_{i_{k}} V\left(x\left(t_{k}\right)\right),
\end{aligned}
$$

since for any positive definite $P \in \mathbb{R}^{n \times n}$ and symmetric $Q \in \mathbb{R}^{n \times n}, x^{T} Q x \leq$ $\lambda_{\max }\left[P^{-1} Q\right] x^{T} P x$. Apply (3.11) and (3.12) successively on each interval. Let 
$V\left(x\left(t_{0}\right)\right)=V_{0}$, then for $t \in\left(t_{0}, t_{1}\right], V(x(t)) \leq V_{0} \exp \left[-\epsilon\left(t-t_{0}\right)\right]$. For $t \in\left(t_{1}, t_{2}\right]$,

$$
\begin{aligned}
V(x(t)) & \leq V\left(x\left(t_{1}^{+}\right)\right) \exp \left[-\epsilon\left(t-t_{1}\right)\right], \\
& \leq \mu_{i_{1}} V\left(x\left(t_{1}\right)\right) \exp \left[-\epsilon\left(t-t_{1}\right)\right], \\
& \leq \mu_{i_{1}}\left(V_{0} \exp \left[-\epsilon\left(t_{1}-t_{0}\right)\right]\right) \exp \left[-\epsilon\left(t-t_{1}\right)\right], \\
& =\mu_{i_{1}} V_{0} \exp \left[-\epsilon\left(t-t_{0}\right)\right] .
\end{aligned}
$$

In general, for $t \in\left(t_{k-1}, t_{k}\right], V(x(t)) \leq V_{0}\left[\Pi_{j=1}^{k-1} \mu_{i_{j}}\right] \exp \left[-\epsilon\left(t-t_{0}\right)\right]$. Without loss of generality, assume that $\Pi_{j=1}^{k-1} \mu_{i_{j}}=\Pi_{j=1}^{k^{+}} \mu_{i_{j}}^{+} \Pi_{j=1}^{k^{-}} \mu_{i_{j}}^{-}$, with $0 \leq \mu_{i_{j}}^{-} \leq 1, \mu_{i_{j}}^{+}>1$, and $k^{+}+k^{-}=k-1$. Note that for $a_{n} \geq 0$, the infinite product satisfies $\Pi_{n=1}^{N}\left(1+a_{n}\right) \leq$ $\exp \left[\sum_{n=1}^{N} a_{n}\right]$. Hence, $V(x(t)) \leq V_{0}\left[\Pi_{j=1}^{k^{-}} \mu_{i_{j}}^{-}\right] \exp \left[-\epsilon\left(t-t_{0}\right)+\sum_{j=1}^{k-1}\left(\mu_{i_{j}}^{+}-1\right)\right]$. This implies that $V(x(t)) \leq V_{0} D\left[\Pi_{j=1}^{k^{-}} \mu_{i_{j}}^{-}\right] \exp \left[-\epsilon\left(t-t_{0}\right)\right]$, and hence the trivial solution is globally exponentially stable under the asynchronous switching rule (3.7)-(3.9).

The switching rule algorithm (3.7)-(3.9) is motivated by the switching rule constructions in $[13,16]$. Note that if there are two or more arguments which minimize $f\left(x_{1}\right)$ for some $x_{1}$, for example if $x_{1}^{T} \Upsilon_{i_{1}} x_{1}=\cdots=x_{1}^{T} \Upsilon_{i_{r}} x_{1}$, then any of $i_{1}, \ldots, i_{r}$ can be chosen for the next switch (for completeness, assume that $f(x)$ select the smallest index, $\left.i=\min \left\{i_{1}, \ldots, i_{r}\right\}\right)$. In the case that the switching rule is arbitrary, sufficient conditions for stabilizability become stricter.

Corollary 3.2. Assume that there exists matrices $G=G^{T}, H_{i}=H_{i}^{T}, K_{i}=K_{i}^{T}$, positive definite matrix $P=P^{T}$, and constants $\mu_{i}$ such that (3.3)-(3.6) hold for $x \in \mathbb{R}^{n}, t \geq t_{0}, i=1,2, \ldots, m$. Assume that $\lambda_{\max }\left[P^{-1} \Upsilon_{i}\right]<0$, where $\Upsilon_{i}=$ $\left(A+B L_{i}\right)^{T} P+P\left(A+B L_{i}\right)+H_{i}+G$, for $i=1,2 \ldots, m$, and that there exists $D>0$ such that $\sum_{k=1}^{\infty}\left(\mu_{i_{k}}-1\right)<D$. Then, the trivial solution of system (3.2) is globally exponentially stable under an arbitrary asynchronous switching rule.

Proof. Consider the common Lyapunov function $V=x^{T} P x$, then it follows from equation (3.10) that,

$$
\dot{V}(x(t)) \leq x^{T} \Upsilon_{i_{k}} x \leq \lambda_{\max }\left[P^{-1} \Upsilon_{i_{k}}\right] V(x(t)),
$$

for $t \neq t_{k}$. Hence, there exists $\epsilon>0$ such that $\dot{V}(x(t)) \leq-\epsilon V(x(t))$, then it follows from the proof of Theorem 3.1 that the trivial solution is globally exponentially stable under arbitrary asynchronous switching.

In Theorem 3.1, it is possible for some of the matrices $\Upsilon_{i}$ to be unstable (at least one eigenvalue has positive real part), which corresponds to growth of the system state in certain subregions of $\mathbb{R}^{n}$. However, since a convex combination of the matrices $\Upsilon_{i}$ is negative definite, in any region in $\mathbb{R}^{n}$ there exists at least one mode such that $\|x(t)\|$ decays. In order to exploit this characteristic, the asynchronous switching rule $\sigma(x)$ must follow the index rule (3.7)-(3.9) outlined above. On the other hand, Corollary 3.2 requires stronger conditions, specifically, that $P^{-1} \Upsilon_{i}$ are Hurwitz matrices (all eigenvalues have negative real part), but ensures stability for arbitrary asynchronous switching and is easier to verify. 


\section{SynChronous CONTROLlER SWitching}

Consider system (2.2) and suppose that the system can switch only at prespecified times $t=k h, k=1,2, \ldots$, where $h>0$ is the switching interval time. More specifically, the switching rule maps the interval to a certain controller at every switching time [7]:

$$
\sigma_{k}:((k-1) h, k h] \rightarrow\{1,2, \ldots, m\}
$$

This is called synchronous controller switching. Note that although the switching times are not state-dependent, the switching rule $\sigma$ may be based on the current state of the system at the time $k h$ (for example, $x(k h)-x(0)$ [7]). Assume that $F(t, x) \equiv 0, J_{i}(t, x) \equiv 0$, and $Q_{i}(t, x) \equiv 0$ for all $x \in \mathbb{R}^{n}, t \geq 0$ (assume that $t_{0}=0$, without loss of generality), $i=1,2, \ldots, m$, then the system is,

$$
\left\{\begin{aligned}
\dot{x} & =\left(A+B L_{i_{k}}\right) x, & & t \in((k-1) h, k h], \\
\Delta x & =C M_{i_{k}} x, & & t=k h, \\
x\left(t_{0}^{+}\right) & =x_{0}, & & k=1,2, \ldots
\end{aligned}\right.
$$

where $i_{k} \in\{1,2, \ldots, m\}$ follows a synchronous switching rule $\sigma_{k}$. Since the impulsive switched system is linear, define $\phi_{i}:=\exp \left(\left(A+B L_{i}\right) h\right)$ as the state transition matrix under switched controller i between the times $t_{1}$ and $t_{2}=t_{1}+h$. That is, $x(k h)=\phi_{i} x\left((k-1) h^{+}\right)$. Hence,

$$
\gamma=\max _{i=1,2, \ldots, n} \exp \left[\left(A+B L_{i}\right) h\right]
$$

is a bound on the growth of the switched system, in between impulses, due to the switched linear controller.

Suppose that there exists a positive definite matrix $P=P^{T}$ and $\epsilon>0$ such that the Lyapunov function $V=x^{T} P x$ satisfies,

$$
V\left(x\left(k h^{+}\right)\right)-V\left(x\left((k-1) h^{+}\right)\right) \leq-\epsilon\left\|x\left((k-1) h^{+}\right)\right\|^{2},
$$

along solutions of (4.2) for $k=1,2, \ldots$, then system (4.2) is said to be quadratically stabilizable via synchronous controller switching [7]. This condition ensures that the impulsive effects combined with the switching controller results in a decrease of the Lyapunov function at the switch times $t=k h$.

Using the growth bound (4.3) and condition (4.4), it follows from the proof of Theorem 2.5.2 in [14] (using $w\left(t_{i_{k+1}}^{+}\right) \leq L w\left(t_{i_{k}}^{+}\right)$instead of $w\left(t_{i_{k+1}}\right) \leq L w\left(t_{i_{k}}\right)$ in the proof's comparison system) that quadratic stabilizability via synchronous controller switching implies global asymptotic stability of the trivial solution. From this, it is possible to extend the results of [7] to include impulsive control with synchronous switching.

Theorem 4.1. Assume that there exists a positive definite matrix $P=P^{T}$ and constants $\tau_{1} \geq 0, \ldots, \tau_{m} \geq 0$ such that $\sum_{i=1}^{m} \tau_{i}=1$ and $\sum_{i=1}^{m} \tau_{i} \Psi_{i}<0$, where $\Psi_{i}=\phi_{i}^{T}\left(I+C M_{i}\right)^{T} P\left(I+C M_{i}\right) \phi_{i}-P$. Define the switching regions $\Omega_{i}=\{x \in$ $\left.\mathbb{R}^{n}: x^{T} \Psi_{i} x \leq x^{T} \Psi_{j} x, j=1,2, \ldots, m\right\}$ and define the minimum rule $f(x)=$ 
$\arg \min _{i} x^{T} \Psi_{i} x$. If the synchronous switching rule follows the algorithm,

(i) set $\sigma=f\left(x\left(t_{0}\right)\right)$ for $t_{0} \leq t \leq t_{0}+h$;

(ii) apply an impulse and let $t_{0}=t_{0}+h^{+}$then go to step (4.5);

then the trivial solution of system (4.2) is globally asymptotically stable.

Proof. Consider the common Lyapunov function $V=x^{T} P x$, then along solutions of system (4.2),

$$
\begin{aligned}
V\left(x\left(k h^{+}\right)\right) & =x^{T}\left(k h^{+}\right) P x\left(k h^{+}\right), \\
& =\left(\left(I+C M_{i_{k}}\right) x(k h)\right)^{T} P\left(\left(I+C M_{i_{k}}\right) x(k h)\right), \\
& =\left(\left(I+C M_{i_{k}}\right) \phi_{i_{k}} x\left((k-1) h^{+}\right)\right)^{T} P\left(\left(I+C M_{i_{k}}\right) \phi_{i_{k}} x\left((k-1) h^{+}\right)\right), \\
& =x^{T}\left((k-1) h^{+}\right) \phi_{i_{k}}^{T}\left(I+C M_{i_{k}}\right)^{T} P\left(I+C M_{i_{k}}\right) \phi_{i_{k}} x\left((k-1) h^{+}\right) .
\end{aligned}
$$

It follows that,

$$
\begin{aligned}
& V\left(x\left(k h^{+}\right)\right)-V\left(x\left((k-1) h^{+}\right)\right) \\
& =x^{T}\left((k-1) h^{+}\right) \phi_{i_{k}}^{T}\left(I+C M_{i_{k}}\right)^{T} P\left(I+C M_{i_{k}}\right) \phi_{i_{k}} x\left((k-1) h^{+}\right) \\
& \quad-x^{T}\left((k-1) h^{+}\right) P x\left((k-1) h^{+}\right), \\
& =x^{T}\left((k-1) h^{+}\right) \Psi_{i_{k}} x\left((k-1) h^{+}\right) .
\end{aligned}
$$

The condition $\tau_{1} x^{T} \Psi_{1} x+\ldots+\tau_{m} x^{T} \Psi_{m} x<0$ implies that at the times $t=$ $(k-1) h^{+}$immediately after the impulse, for any $x \in \mathbb{R}^{n} \backslash\{0\}$, there exists an index $i \in\{1, \ldots, m\}$ such that $x^{T} \Psi_{i} x<0$. Following [7], define $\alpha(x):=\min _{\forall i} x^{T} \Upsilon_{i} x$ for $x \neq 0$ and $\alpha_{0}:=\max _{x:\|x\|=1} \alpha(x)$, which is achieved on this compact set since $\alpha(x)$ is continuous. Since there exists an $i$ for any $x \in \mathbb{R}^{n} \backslash\{0\}$ such that $x^{T} \Psi_{i} x<0$, it follows that $\alpha(x)<0$ for all $x \neq 0$, and hence $\alpha_{0}<0$. Since the switching rule follows (4.5)-(4.6), then it follows from (4.7) that $V\left(x\left(k h^{+}\right)\right)-V\left(x\left((k-1) h^{+}\right)\right) \leq$ $-\epsilon\left\|x\left((k-1) h^{+}\right)\right\|^{2}$ with $\alpha_{0}=-\epsilon<0$. Thus, system (4.2) is quadratically stabilizable under the synchronous switching rule (4.5)-(4.6), and so the trivial solution is globally asymptotically stable.

Immediately after each impulse $t=(k-1) h^{+}$, the switching rule $\sigma$ for the next interval $((k-1) h, k h]$ is determined by evaluating the minimum rule $f(x((k-$ 1) $\left.h^{+}\right)$), which is the index that minimizes $x^{T} \Psi_{i} x$. Again the switching rule algorithm (4.5)-(4.6) is motivated by those found in [13, 16]. As in Section 3, if $f\left(x_{1}\right)$ is minimized by multiple indices $i_{1}, \ldots, i_{r}$ for some $x_{1}$, select the smallest index $i=\min \left\{i_{1}, \ldots, i_{r}\right\}$. To prove stability for arbitrary switching, more strict criteria need to be imposed.

Corollary 4.2. Assume that there exists a positive definite matrix $P=P^{T}$ such that $\lambda_{\max }\left[\Psi_{i}\right]<0$, where $\Psi_{i}=\phi_{i}^{T}\left(I+C M_{i}\right)^{T} P\left(I+C M_{i}\right) \phi_{i}^{T}-P$, for $i=1,2, \ldots, m$, then the trivial solution of system (4.2) is globally asymptotically stable under arbitrary synchronous switching. 
Proof. Consider the common Lyapunov function $V=x^{T} P x$, and begin from equation (4.7),

$$
\begin{aligned}
V\left(x\left(k h^{+}\right)\right)-V\left(x\left((k-1) h^{+}\right)\right) & \leq x^{T}\left((k-1) h^{+}\right) \Psi_{i_{k}} x\left((k-1) h^{+}\right), \\
& \leq \lambda_{\max }\left[\Psi_{i_{k}}\right] x^{T}\left((k-1) h^{+}\right) x\left((k-1) h^{+}\right), \\
& =-\epsilon\left\|x\left((k-1) h^{+}\right)\right\|^{2},
\end{aligned}
$$

where $\epsilon=-\lambda_{\max }\left[\Psi_{i}\right]>0$. Hence, system (4.2) is quadratically stabilizable under any synchronous switching rule, and so the trivial solution is globally asymptotically stable.

Note that Theorem 4.1 has less strict criteria since it is not required for every matrix $\Psi_{i}$ to be Hurwitz, instead, only a convex combination is required to be negative definite. The trade-off is that the prespecified switching rule $\sigma$ must be used which follows the index rule (4.5)-(4.6). Corollary 4.2 requires stronger conditions, but is easier to verify and ensures stability for arbitrary synchronous switching.

\section{Average DWEll-time CONTROller SWitching}

Consider a special class of switching rules that satisfy an average dwell-time condition. Let $N_{\sigma}\left(t_{0}, t\right)$ be the number of discontinuities of a switching rule $\sigma$ over $\left[t_{0}, t\right)$ and let $S_{a}\left[\tau_{a}, N_{0}\right]$ denote the set of all switching rules satisfying [9]:

$$
N_{\sigma}\left(t_{0}, t\right) \leq N_{0}+\frac{t-t_{0}}{\tau_{a}}
$$

where $N_{0}>0$ is known as the chatter bound and $\tau_{a}>0$ is called the average dwell-time. If $\sigma \in S_{a}$, the average time spent in each subsystem can be no less than $\tau_{a}>0$, that is, the switch times satisfy $t_{k}-t_{k-1}>\tau_{a}$ on average. This can be seen by noting that $\left(t-t_{0}\right) / N_{\sigma}$ is the average time spent in each subsystem, which arises in the definition of average dwell-time in the limit as $N_{0} \rightarrow 0$. When $N_{0}>0$, the average time spent in each subsystem must be greater than or equal to $\tau_{a}$. Note that a synchronous switching rule (4.1) satisfies a dwell-time condition (for example, with $\tau_{a}=h$ ), and it is also possible, but not necessary, for an asynchronous switching rule (3.1) to satisfy an average dwell-time condition. In the next theorem, some results of [9] are extended to include weakly nonlinear impulsive control and weakly nonlinear switched control.

Theorem 5.1. Assume that there exists continuous functions $\phi_{i}(t), \xi_{i}(t), \zeta_{i}(t)$, constants $\lambda_{i}, \beta_{i}$, and positive definite matrices $P_{i}=P_{i}^{T}$ such that,

$$
\begin{gathered}
2 F^{T}(t, x) P_{i} x \leq \xi_{i}(t) x^{T} P_{i} x, \\
2 J_{i}^{T}(t, x) B^{T} P_{i} x \leq \phi_{i}(t) x^{T} P_{i} x, \\
2 x^{T}\left(I+C M_{i}\right)^{T} C Q_{i}(t, x)+Q_{i}^{T}(t, x) C^{T} C Q_{i}(t, x) \leq \zeta_{i}(t) x^{T} P_{i} x, \\
\lambda_{\max }\left[P_{i}^{-1}\left(\left(A+B L_{i}\right)^{T} P_{i}+P_{i}\left(A+B L_{i}\right)\right)\right]+\xi_{i}(t)+\phi_{i}(t) \leq \lambda_{i}, \\
\lambda_{\max }\left[\left(I+C M_{i}\right)^{T}\left(I+C M_{i}\right)\right]+\zeta_{i}(t) \leq \beta_{i},
\end{gathered}
$$

for $x \in \mathbb{R}^{n}, t \geq t_{0}$, and $i=1,2, \ldots, m$. Assume that, without loss of generality, $\lambda_{1}<0, \ldots, \lambda_{r}<0, \lambda_{r+1} \geq 0, \ldots, \lambda_{m} \geq 0$. Define $\lambda^{-}:=\max _{1 \leq i \leq r}\left\{\lambda_{i}\right\}, \lambda^{+}:=$ 
$\min _{r+1 \leq i \leq m}\left\{\lambda_{i}\right\}, \beta:=\max _{\forall i}\left\{\beta_{i}\right\}, \rho:=\max _{\forall i}\left\{\lambda_{\max }\left[P_{i}\right] / \lambda_{\min }\left[P_{i}\right]\right\}$. Let $T^{-}\left(t_{0}, t\right)$ and $T^{+}\left(t_{0}, t\right)$ be the total activation times in the subsystems corresponding to $\lambda_{1}, \ldots, \lambda_{r}<0$ and $\lambda_{r+1}, \ldots, \lambda_{m} \geq 0$, respectively. If $T^{+}\left(t_{0}, t\right) \leq q T^{-}\left(t_{0}, t\right)$ for all $t \geq t_{0}$, where $q \geq 0$ is a constant, and if any of the following conditions hold:

(i) $0<\rho \beta \leq 1, t_{k}-t_{k-1} \leq \eta$ for $\eta>0$, and $\frac{\ln (\rho \beta)}{\eta}+\lambda^{-}+q \lambda^{+}<0$;

(ii) $\rho \beta>1, t_{k}-t_{k-1} \geq \delta$ for $\delta>0$, and $\frac{\ln (\rho \beta)}{\delta}(1+q)+\lambda^{-}+q \lambda^{+}<0$;

(iii) $\rho \beta>1, \sigma \in S_{a}$ and $\frac{\ln (\rho \beta)}{\tau_{a}}(1+q)+\lambda^{-}+q \lambda^{+}<0$;

then the trivial solution of system (2.2) is globally exponentially stable.

Proof. As in [9], consider the switched Lyapunov function $V_{i_{k}}=x^{T} P_{i_{k}} x$ where $i_{k} \in\{1,2, \ldots, m\}$ follows the switching rule $\sigma$. For $t \in\left(t_{k-1}, t_{k}\right]$,

$$
\begin{aligned}
\dot{V}_{i_{k}}(x(t))= & {\left[\left(A+B L_{i_{k}}\right) x+F(t, x)+B J_{i_{k}}(t, x)\right]^{T} P_{i_{k}} x } \\
& +x^{T} P_{i_{k}}\left[\left(A+B L_{i_{k}}\right) x+F(t, x)+B J_{i_{k}}(t, x)\right], \\
= & x^{T}\left[\left(A+B L_{i_{k}} x\right)^{T} P_{i_{k}}+P_{i_{k}}\left(A+B L_{i_{k}} x\right)\right] x \\
& +J_{i_{k}}^{T}(t, x) B^{T} P_{i_{k}} x+x^{T} P_{i_{k}} B J_{i_{k}}(t, x) \\
& +F^{T}(t, x) P_{i_{k}} x+x^{T} P_{i_{k}} F(t, x), \\
\leq & \left\{\lambda_{\max }\left[P_{i_{k}}{ }^{-1}\left(\left(A+B L_{i_{k}}\right)^{T} P_{i_{k}}+P_{i_{k}}\left(A+B L_{i_{k}}\right)\right)\right\} x^{T} P_{i_{k}} x\right. \\
& +\left(\xi_{i}(t)+\phi_{i}(t)\right) x^{T} P_{i_{k}} x, \\
\leq & \lambda_{i_{k}} V_{i_{k}}(x(t)) .
\end{aligned}
$$

This implies that,

$$
V_{i_{k}}(x(t)) \leq V_{i_{k}}\left(x\left(t_{k-1}^{+}\right)\right) \exp \left[\lambda_{i_{k}}\left(t-t_{k-1}\right)\right],
$$

from which it follows that,

$$
\lambda_{\min }\left[P_{i_{k}}\right] x^{T}(t) x(t) \leq \lambda_{\max }\left[P_{i_{k}}\right] x^{T}\left(t_{k-1}{ }^{+}\right) x\left(t_{k-1}{ }^{+}\right) \exp \left[\lambda_{i_{k}}\left(t-t_{k-1}\right)\right],
$$

and thus,

$$
\|x(t)\|^{2} \leq \rho\left\|x\left(t_{k-1}^{+}\right)\right\|^{2} \exp \left[\lambda_{i_{k}}\left(t-t_{k-1}\right)\right] .
$$

Equation (5.7) is a bound based on the effects of the switching control. After each time $t=t_{k}$, an impulse is applied:

$$
\begin{aligned}
\left\|x\left(t_{k}^{+}\right)\right\|^{2}= & {\left[\left(I+C M_{i_{k}}\right) x\left(t_{k}\right)+C Q_{i_{k}}\left(t_{k}, x\left(t_{k}\right)\right)\right]^{T}\left[\left(I+C M_{i_{k}}\right) x\left(t_{k}\right)\right.} \\
& \left.+C Q_{i_{k}}\left(t_{k}, x\left(t_{k}\right)\right)\right] \\
= & x^{T}\left(t_{k}\right)\left[\left(I+C M_{i_{k}}\right)^{T}\left(I+C M_{i_{k}}\right)\right] x\left(t_{k}\right) \\
& +x^{T}\left(t_{k}\right)\left(I+C M_{i_{k}}\right)^{T} C Q_{i_{k}}\left(t_{k}, x\left(t_{k}\right)\right) \\
& +Q_{i_{k}}^{T}\left(t_{k}, x\left(t_{k}\right)\right) C^{T}\left(I+C M_{i_{k}}\right) x\left(t_{k}\right) \\
& +Q_{i_{k}}^{T}\left(t_{k}, x\left(t_{k}\right)\right) C^{T} C Q_{i_{k}}\left(t_{k}, x\left(t_{k}\right)\right) \\
\leq & \beta_{i_{k}}\left\|x\left(t_{k}\right)\right\|^{2}
\end{aligned}
$$

Apply (5.7) and (5.8) successively on each subinterval as in the proof of Theorem 1 in [9], and the rest of the proof follows for the three cases. 
Intuitively, in case (i) the impulsive control is a stabilizing force and is applied often enough so as to counteract any growth in $\|x(t)\|$ between switching times. Cases (ii) and (iii) represent the scenarios where the impulses are a destabilizing force, but the system is stabilized by the switching control and by guaranteeing the impulses are not applied too often.

\section{EXAMPLES}

Example 6.1. Consider system (3.2) with $i_{k} \in\{1,2\}, t_{0}=0$,

$$
\begin{gathered}
A=\left(\begin{array}{cc}
0 & 1 \\
-1.25 & 1
\end{array}\right), \quad B=\left(\begin{array}{l}
0 \\
1
\end{array}\right), \quad C=\left(\begin{array}{ll}
1 & 0 \\
0 & 1
\end{array}\right) . \\
F(t, x)=\left(\begin{array}{c}
-x^{2} y-7 / 6 x y^{2} \\
19 / 8 x^{2} y+x y^{2}
\end{array}\right) .
\end{gathered}
$$

Consider the switched and impulsive controllers,

$$
\begin{gathered}
L_{1}^{T}=\left(\begin{array}{c}
3 \\
-6
\end{array}\right), \quad L_{2}^{T}=\left(\begin{array}{c}
-2 \\
1
\end{array}\right), \quad M_{1}=\left(\begin{array}{cc}
-0.4 & 0.1 \\
0.1 & -0.5
\end{array}\right), \quad M_{2}=\left(\begin{array}{cc}
-0.3 & 0 \\
0 & -0.4
\end{array}\right), \\
J_{1}(t, x)=-0.5 \operatorname{sign}(y)|x|, \quad J_{2}(t, x)=0, \\
Q_{1}(t, x)=\left(\begin{array}{c}
-0.25 \operatorname{sign}(x)|y| \\
0
\end{array}\right), \quad Q_{2}(t, x)=0,
\end{gathered}
$$

where,

$$
\operatorname{sign}(y):= \begin{cases}1, & \text { for } y>0 \\ 0, & \text { for } y=0 \\ -1, & \text { for } y<0\end{cases}
$$

The constant matrices $A, B, C, L_{1}, L_{2}$ are taken from [7]. The matrices $A+B L_{1}$ and $A+B L_{2}$ have eigenvalues with positive real part, and so both linear controllers lead to unstable subsystems. The impulsive controllers help in stabilizing the system. With the switched and impulsive controllers applied to system (3.2), it becomes,

$$
\left\{\begin{array}{rlrl}
\dot{x} & =y-x^{2} y-7 x y^{2} / 6, & & t \in\left(t_{k-1}, t_{k}\right], \\
\dot{y} & =1.75 x-5 y+19 x^{2} y / 8+x y^{2}-0.5 \operatorname{sign}(y)|x|, & & \\
\Delta x & =-0.4 x+0.1 y-0.25 \operatorname{sign}(x)|y|, & t=t_{k}, \quad k=1,2, \ldots, \\
\Delta y & =0.1 x-0.5 y, & &
\end{array}\right.
$$

for $i=1$, and,

$$
\left\{\begin{aligned}
\dot{x} & =y-x^{2} y-7 x y^{2} / 6, & & \\
\dot{y} & =-3.25 x+2 y+19 x^{2} y / 8+x y^{2}, & & \\
\Delta x & =-0.3 x, & \left.t=t_{k-1}, t_{k}\right] & \\
\Delta y & =-0.4 y, & &
\end{aligned}\right.
$$

for $i=2$. Choose $G=0, H_{2}=0, K_{2}=0, \mu_{1}=0.9042, \mu_{2}=0.5093$,

$$
H_{1}=\left(\begin{array}{cc}
2 / 3 & 7 / 18 \\
7 / 18 & 0
\end{array}\right), \quad K_{1}=\left(\begin{array}{cc}
0 & 0.2542 \\
0.2542 & 0.4262
\end{array}\right), \quad P=\left(\begin{array}{cc}
19 / 12 & 2 / 3 \\
2 / 3 & 7 / 9
\end{array}\right) \text {. }
$$


Then,

$$
\Upsilon_{1}=\left(\begin{array}{cc}
3 & 0 \\
0 & -6.4444
\end{array}\right), \quad \Upsilon_{2}=\left(\begin{array}{cc}
-4.3333 & 0.3889 \\
0.3889 & 4.4444
\end{array}\right)
$$

Take $\tau_{1}=\tau_{2}=0.5$ and $D=0$ then the conditions of Theorem 3.1 are satisfied. Therefore, the trivial solution of system (3.2) is globally exponentially stable under the asynchronous switching rule (3.7)-(3.9) where,

$$
\begin{aligned}
& \Omega_{1}=\left\{(x, y) \in \mathbb{R}^{2}: 3 x^{2}-6.4444 y^{2} \leq-4.3333 x^{2}+0.7778 x y+4.4444 y^{2}\right\}, \\
& \Omega_{2}=\left\{(x, y) \in \mathbb{R}^{2}: 3 x^{2}-6.4444 y^{2}>-4.3333 x^{2}+0.7778 x y+4.4444 y^{2}\right\} .
\end{aligned}
$$

That is, choose $i=1$ whenever $7.3333 x^{2}-0.7778 x y-10.8888 y^{2}<0$, otherwise choose $i=2$. See Figure 2 for a simulation. Note that because $\lambda_{\max }\left[P^{-1} \Psi_{1}\right]=$ 2.0402 and $\lambda_{\max }\left[P^{-1} \Psi_{2}\right]=7.3538$, the stricter conditions of Corollary 3.2 are not satisfied in this example.

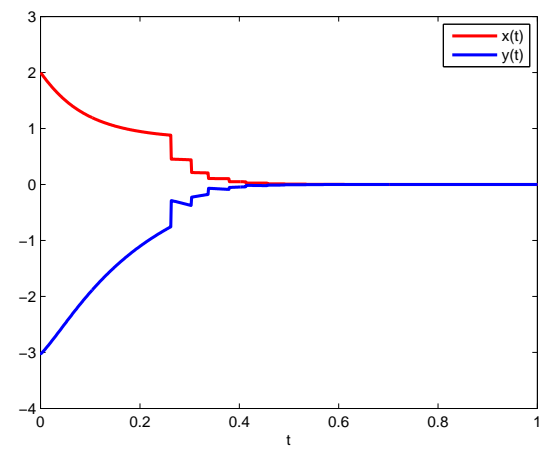

(A) $\left(x_{0}, y_{0}\right)=(2,-3)$

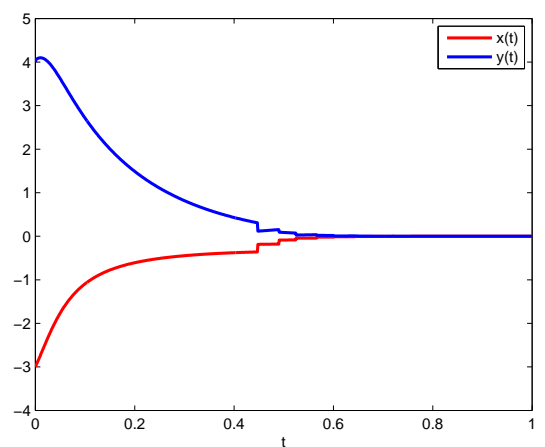

(B) $\left(x_{0}, y_{0}\right)=(-3,4)$

Figure 2. Simulation of Example 6.1.

Example 6.2. Consider system (3.2) with $i_{k} \in\{1,2\}, t_{0}=0$,

$$
\begin{gathered}
A=\left(\begin{array}{cc}
0 & 1 \\
-2.25 & -1
\end{array}\right), \quad B=\left(\begin{array}{l}
0 \\
1
\end{array}\right), \quad C=\left(\begin{array}{ll}
1 & 0 \\
0 & 1
\end{array}\right), \\
F(t, x)=\left(\begin{array}{c}
-x^{2} y-7 / 6 x y^{2} \\
19 / 8 x^{2} y+x y^{2}
\end{array}\right)
\end{gathered}
$$

and switched and impulsive controllers,

$$
\begin{gathered}
L_{1}^{T}=\left(\begin{array}{l}
-1 \\
-6
\end{array}\right), \quad L_{2}^{T}=\left(\begin{array}{c}
-3 \\
-1.5
\end{array}\right), \quad J_{1}(t, x)=-0.5 \operatorname{sign}(y)|x|, \quad J_{2}(t, x)=0, \\
M_{1}=\left(\begin{array}{cc}
0.4 & 0.1 \\
0.1 & 0.5
\end{array}\right), \quad M_{2}=\left(\begin{array}{cc}
0.3 & 0 \\
0 & 0.4
\end{array}\right) \\
Q_{1}(t, x)=H(t-10)\left(\begin{array}{l}
-1.4 x-0.1 y \\
-0.1 x-1.5 y
\end{array}\right) \\
Q_{2}(t, x)=H(t-20)\left(\begin{array}{l}
-1.3 x \\
-1.4 y
\end{array}\right)
\end{gathered}
$$


where $H(t)$ is the Heaviside function. That is, after $t=10$, no impulsive controller is applied when the system is in the first subsystem and crosses a switch threshold. Further, after $t=20$, no impulsive controller is applied when the system is in the second subsystem and crosses a switch threshold. Hence, the impulsive control is switched off for $t \geq 20$. Choose $G=0, K_{1}=0, K_{2}=0, H_{2}=0$,

$$
H_{1}=\left(\begin{array}{cc}
2 / 3 & 7 / 18 \\
7 / 18 & 0
\end{array}\right), \quad P=\left(\begin{array}{cc}
19 / 12 & 2 / 3 \\
2 / 3 & 7 / 9
\end{array}\right)
$$

Then,

$$
\Upsilon_{1}=\left(\begin{array}{ll}
-3.6667 & -5.2222 \\
-5.2222 & -9.5556
\end{array}\right), \quad \Upsilon_{2}=\left(\begin{array}{ll}
-7.0000 & -4.1667 \\
-4.1667 & -2.5556
\end{array}\right)
$$

Hence, $\lambda_{\max }\left[P^{-1} \Psi_{1}\right]=-0.7443$ and $\lambda_{\max }\left[P^{-1} \Psi_{2}\right]=-0.1379$. Take $\mu_{1}=2.5332$, $\mu_{2}=1.9968$, then, since there are a finite number of impulses, say $\nu$, it follows that there exists a $D>0$ such that $\Pi_{k=1}^{\nu}\left(\mu_{i k}-1\right)<D$. Take $\tau_{1}=\tau_{2}=0.5$ then the conditions of Corollary 3.2 are satisfied, and so, the trivial solution of system (3.2) is globally exponentially stable under arbitrary asynchronous switching. See Figure 3 for a simulation with the switching rule $\sigma(x)$ of Example 6.1.

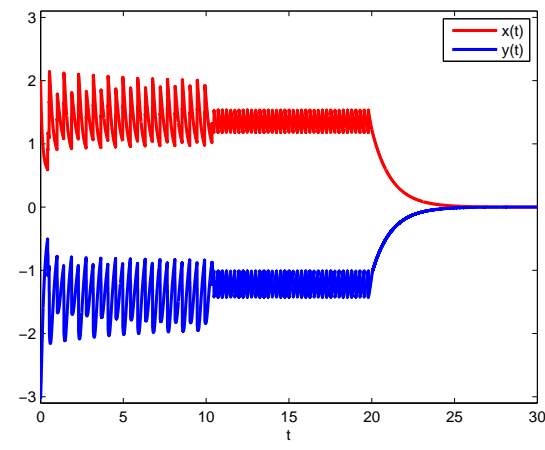

(A) $\left(x_{0}, y_{0}\right)=(2,-3)$

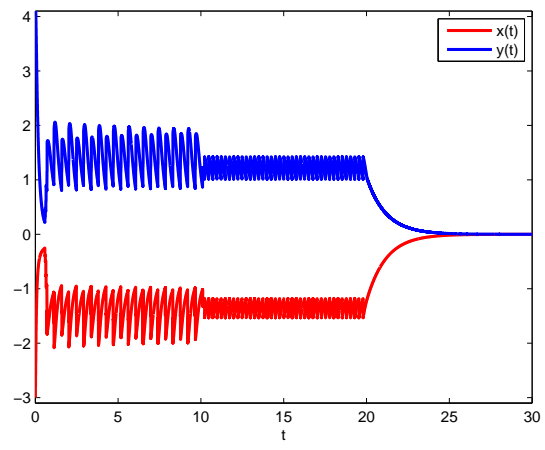

(в) $\left(x_{0}, y_{0}\right)=(-3,4)$

Figure 3. Simulation of Example 6.2.

Example 6.3. Consider system (4.2) with $i_{k} \in\{1,2\}, t_{0}=0$, and $A, B, C, L_{1}$, $L_{2}, M_{1}, M_{2}$ the same as in Example 6.1. Choose $h=0.2$ and,

$$
P=\left(\begin{array}{cc}
19 / 12 & 2 / 3 \\
2 / 3 & 7 / 9
\end{array}\right)
$$

then,

$$
\Psi_{1}=\left(\begin{array}{ll}
-0.7208 & -0.4116 \\
-0.4116 & -0.6920
\end{array}\right), \quad \Psi_{2}=\left(\begin{array}{cc}
-1.1521 & -0.4896 \\
-0.4896 & 0.0115
\end{array}\right) .
$$

If $\tau_{1}=\tau_{2}=0.5$ then all the conditions of Theorem 4.1 are satisfied. Hence, the trivial solution of system (4.2) is globally asymptotically stable under the 
synchronous switching rule (4.5)-(4.6) where,

$$
\begin{gathered}
\Omega_{1}=\left\{(x, y) \in \mathbb{R}^{2}:-0.7208 x^{2}-0.8233 x y-0.6920 y^{2}\right. \\
\left.\leq-1.1521 x^{2}-0.9793 x y+0.0115 y^{2}\right\}, \\
\Omega_{2}=\left\{(x, y) \in \mathbb{R}^{2}:-0.7208 x^{2}-0.8233 x y-0.6920 y^{2}\right. \\
\left.>-1.1521 x^{2}-0.9793 x y+0.0115 y^{2}\right\} .
\end{gathered}
$$

That is, whenever $t=k h^{+}$, evaluate $f(x, y)=0.4313 x^{2}+0.0780 x y-0.7035 y^{2}$, if it is less than zero choose $i=1$, otherwise choose $i=2$. See Figure 4 for a simulation. Note that in this example, it is possible to construct a stabilizing synchronous switching rule as long as the switching interval satisfies $0<h \leq$ 0.2422. This is because the impulses are stabilizing forces, and hence need to be applied often enough to stabilize the system. Since $\lambda_{\max }\left[\Psi_{2}\right]=0.1901$, the more strict conditions of Corollary 4.2 are not satisfied in this example.

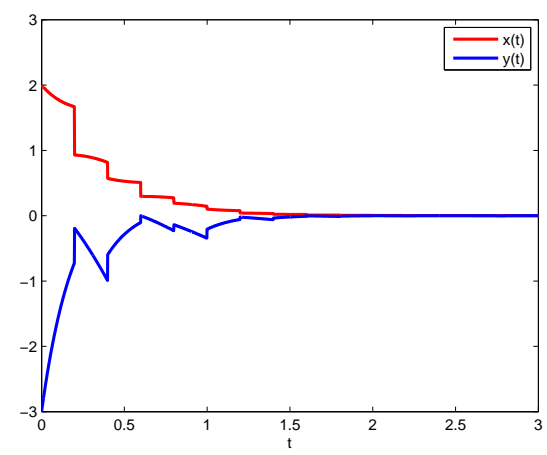

(A) $\left(x_{0}, y_{0}\right)=(2,-3)$

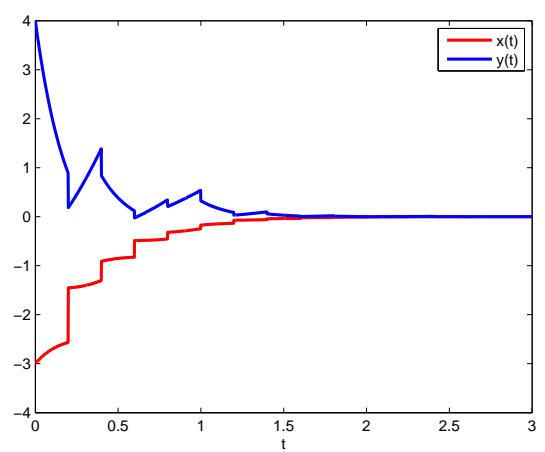

(B) $\left(x_{0}, y_{0}\right)=(-3,4)$

Figure 4. Simulation of Example 6.3.

Example 6.4. Consider system (4.2) with $i_{k} \in\{1,2\}, t_{0}=0$,

$$
A=\left(\begin{array}{cc}
-1 & 0 \\
-1 & -1
\end{array}\right), \quad B=\left(\begin{array}{l}
0 \\
1
\end{array}\right), \quad C=\left(\begin{array}{ll}
1 & 0 \\
0 & 1
\end{array}\right)
$$

and switched and impulsive controllers,

$$
L_{1}^{T}=\left(\begin{array}{c}
1.25 \\
-4
\end{array}\right), \quad L_{2}^{T}=\left(\begin{array}{c}
1.25 \\
-2
\end{array}\right), \quad M_{1}=\left(\begin{array}{cc}
0.1 & 0.1 \\
0.1 & 0.1
\end{array}\right), \quad M_{2}=\left(\begin{array}{cc}
0.3 & 0 \\
0 & 0.4
\end{array}\right) .
$$

Choose the switching interval time $h=0.5$ and,

$$
P=\left(\begin{array}{cc}
19 / 12 & 2 / 3 \\
2 / 3 & 7 / 9
\end{array}\right)
$$

then,

$$
\Psi_{1}=\left(\begin{array}{ll}
-0.7779 & -0.6103 \\
-0.6103 & -0.7703
\end{array}\right), \quad \Psi_{2}=\left(\begin{array}{ll}
-0.5249 & -0.4862 \\
-0.4862 & -0.7019
\end{array}\right)
$$


Hence, $\lambda_{\max }\left[\Psi_{1}\right]=-0.1638$ and $\lambda_{\max }\left[\Psi_{2}\right]=-0.1192$, and so the conditions of Corollary 4.2 are satisfied. Hence, the trivial solution of system (4.2) is globally asymptotically stable under arbitrary synchronous switching. The impulses are destabilizing forces but are not applied too often. See Figure 5 for a simulation with the synchronous switching rule,

$$
\sigma_{k}= \begin{cases}1 & \text { if } t \in(k, k+0.5], k=0,1,2, \ldots \\ 2 & \text { if } t \in(k+0.5, k+1]\end{cases}
$$

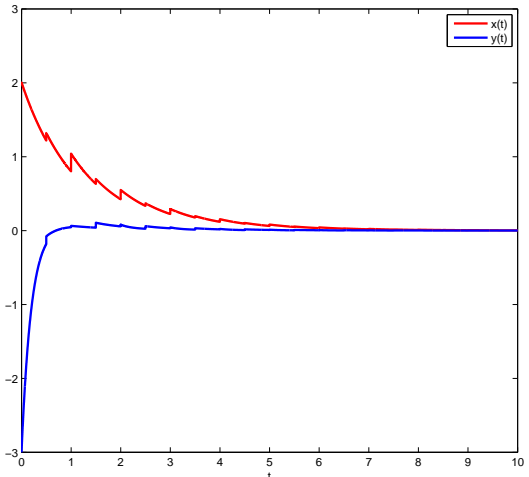

(A) $\left(x_{0}, y_{0}\right)=(2,-3)$

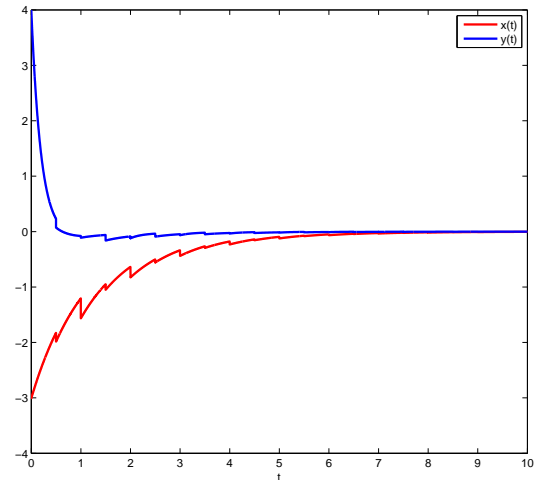

(в) $\left(x_{0}, y_{0}\right)=(-3,4)$

Figure 5. Simulation of Example 6.4.

Example 6.5. Consider system (2.2) with $i_{k} \in\{1,2\}, t_{0}=0$,

$$
\begin{gathered}
A=\left(\begin{array}{cc}
-1 & 0 \\
-1 & -1
\end{array}\right), \quad B=\left(\begin{array}{l}
0 \\
1
\end{array}\right), \quad C=\left(\begin{array}{ll}
1 & 0 \\
0 & 1
\end{array}\right), \\
F(t, x)=\left(\begin{array}{c}
-x^{2} y-7 / 6 x y^{2} \\
19 / 8 x^{2} y+x y^{2}
\end{array}\right)
\end{gathered}
$$

and switched and impulsive controllers,

$$
\begin{gathered}
L_{1}^{T}=\left(\begin{array}{c}
-2 \\
-3.5
\end{array}\right), \quad L_{2}^{T}=\left(\begin{array}{l}
1.25 \\
1.25
\end{array}\right), \quad M_{1}=\left(\begin{array}{cc}
0.2 & 0.1 \\
0.1 & 0.2
\end{array}\right), \quad M_{2}=\left(\begin{array}{cc}
0.3 & 0 \\
0 & 0.4
\end{array}\right), \\
J_{1}(t, x)=-0.5 \operatorname{sign}(y)|x|, \quad J_{2}(t, x)=0, \\
Q_{1}(t, x)=\left(\begin{array}{c}
-0.1 \operatorname{sign}(x)|y| \\
0
\end{array}\right), \quad Q_{2}(t, x)=0 .
\end{gathered}
$$

Note that $A+B L_{1}$ is unstable and $A+B L_{2}$ is stable (all eigenvalues have negative real part), and the impulses are destabilizing forces. Take $\phi_{1}(t)=0.45, \phi_{2}(t)=0$, $\xi_{1}(t)=0, \xi_{2}(t)=0, \zeta_{1}(t)=0.4, \zeta_{2}(t)=0, \lambda_{1}=-1.5500, \lambda_{2}=0.6926, \beta_{1}=$ $2.0900, \beta_{2}=1.9600$,

$$
P_{1}=P_{2}=\left(\begin{array}{cc}
19 / 12 & 2 / 3 \\
2 / 3 & 7 / 9
\end{array}\right)
$$


Then, (5.2)-(5.6) are satisfied and $\lambda^{-}=-1.5500, \lambda^{+}=0.6926, \beta=2.0900$, $\rho=4.8783$. Assume that the switching rule is,

$$
\sigma_{k}= \begin{cases}1 & \text { if } t \in(5 k, 5 k+1], k=0,1,2, \ldots \\ 2 & \text { if } t \in(5 k+1,5 k+5] .\end{cases}
$$

Take $q=1 / 3, \tau_{a}=2.3470$, then $\rho \beta=10.1957,(1+q) \ln (\rho \beta) / \tau_{a}+\lambda^{-}+q \lambda^{+}=$ -0.0807 , and so the conditions of case (iii) in Theorem 5.1 are satisfied, and hence the trivial solution of system (2.2) is globally exponentially stable. Note that $\tau_{a} \geq 2.4825$ is required for stabilization with these parameters. See Figure 6 for a simulation.

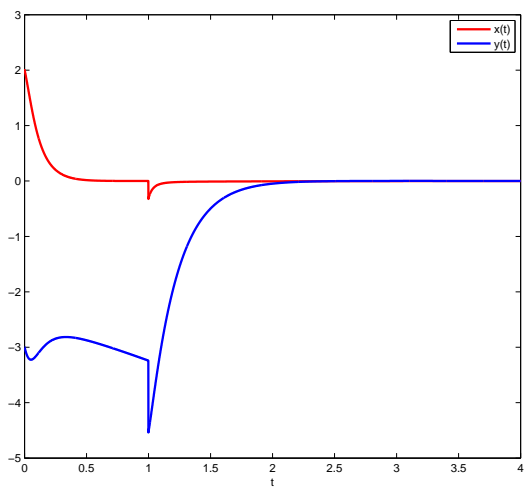

(A) $\left(x_{0}, y_{0}\right)=(2,-3)$

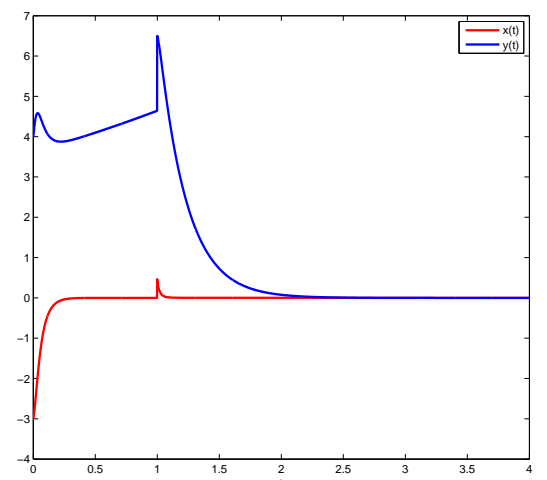

(B) $\left(x_{0}, y_{0}\right)=(-3,4)$

Figure 6. Simulation of Example 6.5.

\section{Conclusion}

In this paper, switching control strategies for a class of switched impulsive nonlinear systems are developed. Both common Lyapunov functions and switched Lyapunov functions are used to give criteria for the stabilizability of systems under switched and impulsive control. First, asynchronous switching is studied and some criteria are established which guarantee the trivial solution is exponentially stable for a prespecified switching rule. Next, some more strict criteria are outlined for the exponential stability of the trivial solution under arbitrary asynchronous switching. Synchronous switching is investigated and sufficient conditions are established for the asymptotic stability of the trivial solution under both arbitrary synchronous switching and a prespecified synchronous switching rule. Finally, some results for average dwell-time satisfying switching are extended to include weakly nonlinear switching controllers and weakly nonlinear impulsive controllers. Examples are given, with simulations using Matlab, to illustrate the theorems developed.

One possible future direction is extending the results for synchronous controller switching to the nonlinear case. Another possibility is to extend the results to switched and impulsive systems which exhibit stronger nonlinearities. 


\section{ACKNOWLEDGEMENTS}

This research was financially supported by the Natural Sciences and Engineering Research Council of Canada (NSERC) as well as the Ontario Graduate Scholarship Program (OGS).

\section{REFERENCES}

[1] A. Bacciotti and L. Mazzi, An invariance principle for nonlinear switched systems, Systems \& Control Letters 54 (2005), no. 11, 1109 - 1119.

[2] M.S. Branicky, Stability of switched and hybrid systems, Proceedings of the 33rd IEEE Conference on Decision and Control 4 (1994), 3498-3503.

[3] _ Multiple lyapunov functions and other analysis tools for switched and hybrid systems, IEEE Transactions on Automatic Control 43 (1998), no. 4, 475-482.

[4] J. Daafouz, P. Riedinger, and C. Iung, Stability analysis and control synthesis for switched systems: A switched lyapunov function approach, IEEE Transactions on Automatic Control 47 (2002), no. 11, 1883-1887.

[5] G. Davrazos and N. T. Koussoulas, A review of stability results for switched and hybrid systems, Proceedings of 9th Mediterranean Conference on Control and Automation (2001).

[6] R. A. Decarlo, M. S. Branicky, S. Pettersson, and B. Lennartson, Perspectives and results on the stability and stabilizability of hybrid systems, Proceedings of the IEEE 88 (2000), no. $7,1069-1082$.

[7] R. J. Evans and A. V. Savkin, Hybrid dynamical systems, Birkhauser, 2002.

[8] Zhi-Hong Guan, D.J. Hill, and Xuemin Shen, On hybrid impulsive and switching systems and application to nonlinear control, IEEE Transactions on Automatic Control 50 (2005), no. $7,1058-1062$.

[9] Zhi-Hong Guan, D.J. Hill, and Jing Yao, A hybrid impulsive and switching control strategy for synchronization of nonlinear systems and application to chua's chaotic circuit, International Journal of Bifurcation and Chaos 16 (2006), no. 1, 229 - 238.

[10] J.P. Hespanha, Extending lasalle's invariance principle to switched linear systems, Proceedings of the 40th IEEE Conference on Decision and Control (2001), 2496-2501.

[11] _ Uniform stability of switched linear systems: Extensions of lasalle's invariance principle, IEEE Transactions on Automatic Control 49 (2004), no. 4, 470-482.

[12] J.P. Hespanha and A.S. Morse, Stability of switched systems with average dwell-time, Proceedings of the 38th IEEE Conference on Decision and Control 3 (1999), 2655-2660.

[13] Sehjeong Kim, S.A. Campbell, and Xinzhi Liu, Stability of a class of linear switching systems with time delay, IEEE Transactions on Circuits and Systems I: Regular Papers 53 (2006), no. 2, $384-393$.

[14] Z. Li, Y. Soh, and C. Wen, Switched and impulsive systems: Analysis, design, and applications, Springer-Verlag, Berlin Heielberg, 2005.

[15] D. Liberzon and A. S. Morse, Basic problems in stability and design of switched systems, IEEE Control Systems Magazine 19 (1999), no. 5, 59 - 70.

[16] Jun Liu, Xinzhi Liu, and Wei-Chau Xie, On the (h0,h)-stabilization of switched nonlinear systems via state-dependent switching rule, Applied Mathematics and Computation 217 (2010), no. 5, $2067-2083$.

[17] Y. Mori, T. Mori, and Y. Kuroe, A solution to the common lyapunov function problem for continuous-time systems, Proceedings of the 36th IEEE Conference on Decision and Control 4 (1997), 3530-3531.

[18] E. Moulay, R. Bourdais, and W. Perruquetti, Stabilization of nonlinear switched systems using control lyapunov functions, Nonlinear analysis: Hybrid Systems 1 (2007), no. 4, 482-490. 
[19] K.S. Narendra and J. Balakrishnan, A common lyapunov function for stable lti systems with commuting a-matrices, IEEE Transactions on Automatic Control 39 (1994), no. 12, 2469-2471.

[20] S. Pettersson and B. Lennartson, Stability and robustness for hybrid systems, Proceedings of the 35th IEEE Conference on Decision and Control 2 (1996).

[21] R.N. Shorten, F.O. Cairbre, and P. Curran, On the dynamic instability of a class of switching system, International Journal of Control 79 (2006), no. 6, 630-635.

[22] Robert Shorten, Fabian Wirth, Oliver Mason, Kai Wulff, and Christopher King, Stability criteria for switched and hybrid systems, SIAM Review 49 (2007), no. 4, 545-592.

[23] A. van der Schaft and H. Schumacher, An introduction to hybrid dynamical systems, Springer-Verlag, London, 2000.

[24] Hui Ye, A.N. Michel, and Ling Hou, Stability theory for hybrid dynamical systems, IEEE Transactions on Automatic Control 43 (1998), no. 4, 461 -474.

1 Department of Applied Mathematics, University of Waterloo, Waterloo, Ontario N2L 3G1, CANada.

E-mail address: xzliu@uwaterloo.ca, xzliu@math.uwaterloo.ca (X. Liu)

E-mail address: pstechli@math.uwaterloo.ca (P. Stechlinski) 